\title{
Effects of ambient temperature and rumen-protected fat supplementation on growth performance, rumen fermentation and blood parameters during cold season in Korean cattle steers
}

\author{
Hyeok Joong Kang ${ }^{1}$, Min Yu Piao ${ }^{1}$, Seung Ju Park', Sang Weon Na ${ }^{1}$, Hyun Jin Kim', and Myunggi Baik ${ }^{1,2, *}$
}

\begin{abstract}
* Corresponding Author: Myunggi Baik Tel: +82-2-880-4809, Fax: +82-2-873-2271, E-mail: mgbaik@snu.ac.kr
\end{abstract}

1 Department of Agricultural Biotechnology and Research Institute of Agriculture and Life Sciences, College of Agriculture and Life Sciences, Seoul National University, Seoul 08826, Korea ${ }^{2}$ Institute of Green Bio Science and Technology, Seoul National University, Pyeongchang 25354, Korea

ORCID

Hyeok Joong Kang

https://orcid.org/0000-0003-0732-1598 Min Yu Piao

https://orcid.org/0000-0002-7067-7741

Seung Ju Park

https://orcid.org/0000-0003-2035-2698

Sang Weon $\mathrm{Na}$

https://orcid.org/0000-0001-5529-653X

Hyun Jin Kim

https://orcid.org/0000-0003-1289-7292

Myunggi Baik

https://orcid.org/0000-0001-8373-9772

Submitted Aug 17, 2018; Revised Oct 8, 2018; Accepted Nov 7, 2018
Objective: This study was performed to evaluate whether cold ambient temperature and dietary rumen-protected fat (RPF) supplementation affect growth performance, rumen fermentation, and blood parameters in Korean cattle steers.

Methods: Twenty Korean cattle steers (body weight [BW], $550.6 \pm 9.14 \mathrm{~kg}$; age, $19.7 \pm 0.13$ months) were divided into a conventional control diet group $(n=10)$ and a $0.5 \%$ RPF supplementation group $(\mathrm{n}=10)$. Steers were fed a concentrate $\operatorname{diet}(1.6 \% \mathrm{BW})$ and a rice straw diet $(1 \mathrm{~kg} / \mathrm{d})$ for 16 weeks (January 9 to February 5 [P1], February 6 to March 5 [P2], March 6 to April 3 [P3], and April 4 to May 2 [P4]).

Results: The mean and minimum indoor ambient temperatures in $\mathrm{P} 1\left(-3.44^{\circ} \mathrm{C},-9.40^{\circ} \mathrm{C}\right)$ were lower $(\mathrm{p}<0.001)$ than those in $\mathrm{P} 3\left(5.87^{\circ} \mathrm{C},-1.86^{\circ} \mathrm{C}\right)$ and $\mathrm{P} 4\left(11.18^{\circ} \mathrm{C}, 4.28^{\circ} \mathrm{C}\right)$. The minimum temperature in $\mathrm{P} 1$ fell within the moderate cold-stress (CS) category, as previously reported for dairy cattle, and the minimum temperatures of $\mathrm{P} 2$ and $\mathrm{P} 3$ were within the mild CS category. Neither month nor RPF supplementation affected the average daily gain or gain-to-feed ratio ( $p>0.05)$. Ruminal ammonia nitrogen concentrations were higher $(\mathrm{p}<0.05)$ in cold winter than spring. Plasma cortisol concentrations were lower $(\mathrm{p}<0.05)$ in the coldest month than in the other months. Serum glucose concentrations were generally higher in colder months than in the other months but were unaffected by RPF supplementation. RPF supplementation increased both total cholesterol $(\mathrm{p}=0.004)$ and high-density lipoprotein (HDL) concentrations $(\mathrm{p}=0.03)$. Conclusion: Korean cattle may not be significantly affected by moderate CS, considering that the growth performance of cattle remained unchanged, although variations in blood parameters were observed among the studied months. RPF supplementation altered cholesterol and HDL concentrations but did not affect growth performance.

Keywords: Ambient Temperature; Beef Cattle; Blood Metabolites; Cold Stress; Growth; Rumen-protected Fat

\section{INTRODUCTION}

Exposure of feedlot beef cattle to cold stress reduces growth performance and feed efficiency due to an increase in the maintenance energy required to retain body temperature [1]. Several studies have reported that cold stress negatively influences mortality, the immune system of calves, back fat thickness, the meat quality of beef cattle, and the milk yield of dairy cows [2-4]. Decreased productivity under cold conditions may result from increased basal metabolic intensity [5]. Moreover, cold stress appears to alter the metabolic and digestive status of animals. For example, increases in circulating glucose and non-esterified fatty acids (NEFAs) are likely triggered by elevated metabolic heat production and mobilization of substrates for energy metabolism in adipose tissue and liver [4]. In addition, increases in rumination activity, reticulorumen motility, and the rate of digesta passage have been observed under cold con- 
ditions [6]. Such changes in rumen digestive characteristics have been associated with reduced digestion in the reticulorumen, particularly when consuming roughage feed [7]. Rumen fermentation characteristics, including volatile fatty acid (VFA) concentrations, are also altered by cold exposure [8]. However, little information on the effects of cold conditions on growth performance, rumen fermentation, and blood parameters is available for Korean cattle.

Dietary fat supplementation has been reported to alleviate cold stress and increase animal productivity [9]. Due to the relatively higher caloric density of fat, dietary fat supplementation in ruminants may provide additional energy, to meet elevated energy requirements under cold conditions [10]. For example, in lactating dairy cows, dietary fat supplementation was associated with increased circulating glucose, which sufficiently increased substrate availability and thus reduced substrate mobilization from energy reserves [11]. Therefore, dietary fat supplementation could represent an effective strategy to alleviate cold stress. However, little information is available on the effects of fat supplementation on alleviating cold stress in Korean cattle. Dietary rumen-protected fat (RPF) has been used as an energy supplement to enhance the productivity of cattle $[12,13]$. Therefore, this study was performed to examine the effects of RPF supplementation on the growth performance, rumen fermentation, and blood parameters of Korean cattle steers under cold conditions.

\section{MATERIALS AND METHODS}

\section{Animals and feeding trial}

All experimental procedures involving animals were approved by the Seoul National University Institutional Animal Care and Use Committee (SNUIACUC), Republic of Korea, and conducted in accordance with the Animal Experimental Guidelines of the SNUIACUC. The study was conducted at the University Animal Farm of the College of Agriculture and Life Sciences, Pyeongchang Campus of Seoul National University, South Korea.

In the feedlot trial, 20 Korean cattle steers with an average age of $19.7 \pm 0.13$ months and body weight (BW) of $550.6 \pm 9.14$ $\mathrm{kg}$ were used. The steers had been fed commercial early fattening stage concentrate using an automatic feeding station (DeLaval Alpro System; DeLaval, Tumba, Sweden) and rice straw, following a conventional feeding program. Water was provided freely. During the 2-week adaptation period before the experiment, all animals were fed an experimental control concentrate (approximately 1.6\% BW/animal) and rice straw $(1 \mathrm{~kg} / \mathrm{d} / \mathrm{head})$. Steers were assigned to one of two treatments: the control group and RPF supplementation group. The RPF is prilled form of palm oil, as described [14], and purchased from Ecolex SDN. BHD (Pulau Indah, Selangor, Malyasia). The RPF was composed of $99.63 \%$ free fatty acids, including
85.48\% palmitic acid (C 16:0), 7.05\% oleic acid (C 18:1), 3.45\% myristic acid (C 14:0), 1.64\% linoleic acid (C 18:2), and 1.04\% lauric acid (C 12:0), with an energy density of $9,316 \mathrm{kcal} / \mathrm{kg}$ (Haneol Corp., Anseong, Korea). Contents of the distillers dried grains with solubles, corn flour, corn gluten feed, barley stone, and palm oil were also adjusted to make similar nonfiber carbohydrate, neutral detergent fiber (NDF), and acid detergent fiber $(\mathrm{ADF})$ between two diets. Table 1 lists the formula and chemical compositions of the experimental diets. Steers were fed a concentrate diet (1.6\% BW) using an automatic feeding station and a rice straw $\operatorname{diet}(1 \mathrm{~kg} / \mathrm{d})$ for 16 weeks (January 9 to February 5 [P1], February 6 to March 5 [P2], March 6 to April 3 [P3], and April 4 to May 2 [P4]). The daily feed intake of the concentrate was automatically recorded online using a computer with the DeLaval Alpro system. Equal amounts of roughage were provided twice daily (08:00 and 18:00) and residual roughage was weighed before the morning feeding. Concentrate and rice straw samples were collected weekly and stored at $-20^{\circ} \mathrm{C}$ until analysis. $\mathrm{BW}$ was measured before morning feeding on the start day, at 4-week intervals thereafter.

\section{Analysis of chemical composition of feed}

The chemical compositions (dry matter, crude protein, ether extract, ash, calcium, and phosphorus) of the concentrate and rice straw were determined using the AOAC method [15]. The $\mathrm{NDF}$ and $\mathrm{ADF}$ contents of the rice straw were analyzed using the sequential method with an ANKOM200 Fiber Analyzer (Ankom Technology Corp., Macedon, NY, USA) and reagents, as described by Van Soest et al [16].

\section{Blood collection and measurement of ambient temperature}

Blood was collected before feeding (after $9 \mathrm{~h}$ of fasting) at approximately 09:00 on the start date, and at 4-week intervals thereafter. Blood was collected via jugular venipuncture with both a non-heparinized vacutainer $(20 \mathrm{~mL}$; Becton-Dickinson, Franklin Lakes, NJ, USA) and ethylenediaminetetraacetic acidtreated vacutainer $(20 \mathrm{~mL})$. Serum and plasma were separated by centrifugation at $1,500 \times g$ at $4^{\circ} \mathrm{C}$ for $15 \mathrm{~min}$ and stored at $-80^{\circ} \mathrm{C}$ until analysis.

Ambient and climate temperatures and relative humidity inside and outside the barn, were recorded in 1-h intervals using four HOBO data loggers (Onset Computer Corp., Bourne, MA, USA). Minimum, mean, and maximum temperatures and corresponding relative humidity data were chosen at every day, and monthly data were average values of 28 days per month. The experimental farm was covered with a roof and the animals were raised indoors; therefore, the animals were protected from precipitation and direct sunlight. Doors were installed on both sides of the barn, which remained open to allow exposure to cold weather. Thus, low winds may have affected 
Table 1. Ingredients of the concentrate and composition of experimental diets for Korean cattle steers

\begin{tabular}{|c|c|c|c|c|c|c|}
\hline Items & Control concentrate & RPF concentrate & & Control concent & trate & RPF concentrate \\
\hline Ingredients (\% DM) & & & \multicolumn{4}{|c|}{ Chemical composition of concentrates (\% DM) } \\
\hline Ground corn & 1.48 & 0.68 & DM & 88.46 & & 88.25 \\
\hline Wheat bran & 25.00 & 25.00 & Moisture & 11.54 & & 11.75 \\
\hline Rice bran & 4.88 & 5.00 & $C P$ & 14.50 & & 14.50 \\
\hline Soy hull & 8.00 & 8.00 & $\mathrm{EE}$ & 3.63 & & 5.30 \\
\hline Urea & 0.39 & 0.35 & Ash & 9.98 & & 7.93 \\
\hline Salt & 0.20 & 0.20 & NDF & 27.26 & & 27.60 \\
\hline Molasses & 3.50 & 3.50 & $A D F$ & 11.54 & & 11.75 \\
\hline Whole cottonseed & 3.00 & 3.00 & NFC & 34.28 & & 34.40 \\
\hline Cottonseed hull & 3.00 & 3.00 & $\mathrm{Ca}$ & 1.45 & & 1.32 \\
\hline Bentonite & 0.30 & 0.30 & $P$ & 0.45 & & 0.46 \\
\hline Magnesium oxide & 0.30 & 0.30 & Crude fiber & 8.93 & & 9.23 \\
\hline Sodium bicarbonate & 0.45 & 0.45 & $\mathrm{ME}^{3)}(\mathrm{Mcal} / \mathrm{kg})$ & 2.47 & & 2.63 \\
\hline Steamed-flaked corn & 21.00 & 21.00 & $\mathrm{NE}^{4)}(\mathrm{Mcal} / \mathrm{kg})$ & 1.12 & & 1.22 \\
\hline Corn flour & 5.48 & 7.61 & \multicolumn{4}{|c|}{ Chemical composition of rice straw (\% DM) } \\
\hline Condensed molasses soluble & 1.20 & 1.20 & DM & \multicolumn{3}{|c|}{74.91} \\
\hline Coconut meal & 5.00 & 5.00 & $C P$ & \multicolumn{3}{|c|}{3.87} \\
\hline Rapeseed meal & 2.41 & 2.04 & $\mathrm{EE}$ & \multicolumn{3}{|c|}{1.37} \\
\hline Wheat flour & 5.00 & 5.00 & Ash & \multicolumn{3}{|c|}{11.46} \\
\hline Distiller's dried grains with solubles & 0.00 & 0.61 & $\mathrm{Ca}$ & \multicolumn{3}{|c|}{0.25} \\
\hline Corn gluten feed & 4.78 & 3.88 & $P$ & \multicolumn{3}{|c|}{0.095} \\
\hline Limestone & 2.89 & 2.57 & $A D F$ & \multicolumn{3}{|c|}{51.97} \\
\hline Live yeast & 0.07 & 0.01 & NDF & \multicolumn{3}{|c|}{31.52} \\
\hline Barley stone & 1.18 & 0.00 & & & & \\
\hline Rumen protected fat & 0.00 & 0.50 & & & & \\
\hline Palm oil & 0.30 & 0.61 & & & & \\
\hline Mineral/vitamin premix ${ }^{1)}$ & 0.19 & 0.19 & & & & \\
\hline Total & 100.00 & 100.00 & & & & \\
\hline $\mathrm{TDN}^{2)}$ & 71.79 & 75.36 & & & & \\
\hline
\end{tabular}

DM, dry matter; RPF, rumen-protected fat; TDN, total digestible nutrients; CP, crude protein; EE, ether extract; NDF, neutral detergent fiber; ADF, acid detergent fiber; NFC, non-fiber carbohydrate; $\mathrm{ME}$, metabolizable energy; $\mathrm{NE}$, net energy.

1) Mineral and vitamin premix contained vit A 2,650,000 IU, vit D 530,000 IU, vit E 1,050 IU, niacin 10,000 mg, Mn 4,400 mg, Zn 4,400 mg, Fe 13,200 mg, Cu 2,200 mg, iodine $440 \mathrm{mg}$, and Co $440 \mathrm{mg} / \mathrm{kg}$ of Grobic-DC. Grobic-DC was provided by Bayer Health Care (Leverkusen, Germany).

${ }^{2)} \mathrm{TDN}(\%)=\mathrm{NFC}+\mathrm{CP}+[(\mathrm{EE}-1) \times 2.25]+\mathrm{NDF}-7[10]$.

3) $\mathrm{ME}=[1.01 \times(\mathrm{DE})-0.45]+0.0046 \times(\mathrm{EE}-3)[10]$.

4) $\mathrm{NE}=1.42 \mathrm{ME}-0.174 \mathrm{ME}^{2}+0.0122 \mathrm{ME}^{3}-1.65[10]$.

the wind-chill temperature.

\section{Rumen fluid collection and analysis}

After blood collection, rumen fluid was collected before feeding (after $9 \mathrm{~h}$ of fasting) using the oral stomach tube method, as described by Shen et al [17]. Rumen fluid $\mathrm{pH}$ was measured immediately with a $\mathrm{pH}$ meter (Ohaus Corp., Parsippany, NJ, USA). For the VFA analysis, $1 \mathrm{~mL}$ of rumen fluid was mixed with $0.2 \mathrm{~mL}$ of $25 \%$ meta-phosphoric acid and stored at $-20^{\circ} \mathrm{C}$ until analysis, and an additional $30 \mathrm{~mL}$ of rumen fluid was stored at $-20^{\circ} \mathrm{C}$ for the ruminal ammonia nitrogen $\left(\mathrm{NH}_{3}-\mathrm{N}\right)$ analysis. $\mathrm{NH}_{3}-\mathrm{N}$ concentrations were determined using a modified colorimetric method [18]. VFA concentrations were determined by gas chromatography using an Agilent Tech 7890A (Hewlett Packard, Waldbronn, Germany) with a Supelco fused silica capillary column $(30 \mathrm{~m} \times 0.25 \mathrm{~mm} \times 0.25 \mu \mathrm{m})$.

\section{Blood analysis}

The analytical reagents for albumin, glucose, triglycerides (TG), high-density lipoprotein (HDL), low-density lipoprotein (LDL), cholesterol, glutamic oxaloacetic transaminase (GOT), glutamic pyruvate transaminase (GPT), calcium, magnesium, and phosphorus were purchased from JW Medical (Seoul, Korea). The analytical reagents for the NEFA analysis were purchased from Wako Pure Chemical (Osaka, Japan). These parameters were analyzed using an automated chemistry analyzer (7180; Hitachi, Tokyo, Japan). Plasma cortisol was analyzed using a Cortisol Salivary HS Enzyme-linked Immunosorbent Assay Kit (cat. no. SLV4635; DRG Instruments, Marburg, Germany). The intra- and inter-assay coefficients of variation for the cortisol kit were $4.0 \%$ and $4.6 \%$, respectively, based on bovine plasma samples. All analytical methods were verified in our laboratory, as reported previously [19]. 


\section{Statistical analysis}

Differences in climate data by month were analyzed with oneway analysis of variance (ANOVA). Differences in growth performance, blood parameters, and rumen fluid parameters by month and dietary treatment were analyzed using a repeatedmeasures two-way ANOVA. The statistical model included month, diet, and their interaction. A p value less than 0.05 was considered to indicate significance. All statistical tests were performed using R Studio for Windows software (R Studio, Boston, MA, USA).

\section{RESULTS AND DISCUSSION}

\section{Climate conditions}

The mean $\left(\mathrm{P} 1:-3.44^{\circ} \mathrm{C}, \mathrm{P} 2:-0.70^{\circ} \mathrm{C}\right)$ and minimum $\left(\mathrm{P} 1:-9.40^{\circ} \mathrm{C}\right.$, $\left.\mathrm{P} 2:-5.5^{\circ} \mathrm{C}\right)$ indoor ambient temperatures in January $(\mathrm{P} 1)$ and February $(\mathrm{P} 2)$ were lower $(\mathrm{p}<0.001)$ than those in April (P4; mean: $11.18^{\circ} \mathrm{C}$, minimum: $4.28^{\circ} \mathrm{C}$; Table 2 ). In a previous study, cold stress was categorized as mild $\left(0^{\circ} \mathrm{C}\right.$ to $\left.-6.7^{\circ} \mathrm{C}\right)$, moderate $\left(-7.2^{\circ} \mathrm{C}\right.$ to $\left.-13.9^{\circ} \mathrm{C}\right)$, or severe $\left(<-13.9^{\circ} \mathrm{C}\right)$ under drywinter-coat conditions in cattle [20]. Therefore, the minimum temperatures of $\mathrm{P} 1$ and both $\mathrm{P} 2$ and $\mathrm{P} 3$ in this study were considered to represent moderate and mild cold-stress (CS) conditions, respectively. In addition, we measured the average temperature during blood collection (08:00 to 10:00). The temperatures were $-6.42^{\circ} \mathrm{C},-0.29^{\circ} \mathrm{C}, 6.79^{\circ} \mathrm{C}, 10.8^{\circ} \mathrm{C}$, and $14.0^{\circ} \mathrm{C}$ on January 9, February 6, March 6, April 4, and May 2, respectively. Then sampling days in January and February were classified as mild CS conditions, while sampling days in March, April and May were considered as thermo-neutral conditions.

\section{Growth performance}

The BW was higher $(\mathrm{p}<0.001)$ during P4 than P1, reflecting animal age. Moreover, the daily concentrate intake was higher $(\mathrm{p}=0.03)$ during P4 than P1 (Table 3). In this study, the daily allowance of concentrate was set at $1.6 \% \mathrm{BW}$ and was adjusted each month based on BW. Thus, the higher concentrate intake during $\mathrm{P} 4$ reflected the increased concentrate allowance, corresponding to a higher BW during P4 than P1. Daily forage intake was higher in P1 than in the other months. RPF supplementation did not affect concentrate and roughage intake during all studied months. Neither month nor RPF supplementation affected the average daily gain and feed efficiency (gain-to-feed ratio; Table 3). In a previous study, dietary RPF supplementation ( $4.5 \%$ fatty acid calcium salts) increased the G:F ratio in beef cattle [13]. In South Korea, commercial feed for Korean cattle steers has generally higher energy levels to produce highly marbled beef [21]. Additional energy supply through RPF supplementation may not have significant impact on growth performance in mild cold conditions. Another possible explanation for no influence on growth performance by fat addition is an insufficient amount of RPF supplementation $(0.5 \%)$.

\section{Rumen volatile fatty acids and $\mathrm{NH}_{3}-\mathrm{N}$}

Neither month nor RPF supplementation affected ruminal $\mathrm{pH}$ ( $\mathrm{p}>0.05$; Table 4). However, ruminal $\mathrm{NH}_{3}-\mathrm{N}$ concentrations were higher $(\mathrm{p}<0.05)$ in cold winter (February) than spring (April and May) (Table 4). For comparison, in sheep, $\mathrm{NH}_{3}$ production was dependent on diet and ambient temperature [22]. Moreover, cold exposure did not affect ruminal

Table 2. Mean, maximum, and minimum values of ambient temperatures, climate temperatures, and relative humidity during January through April of 2015

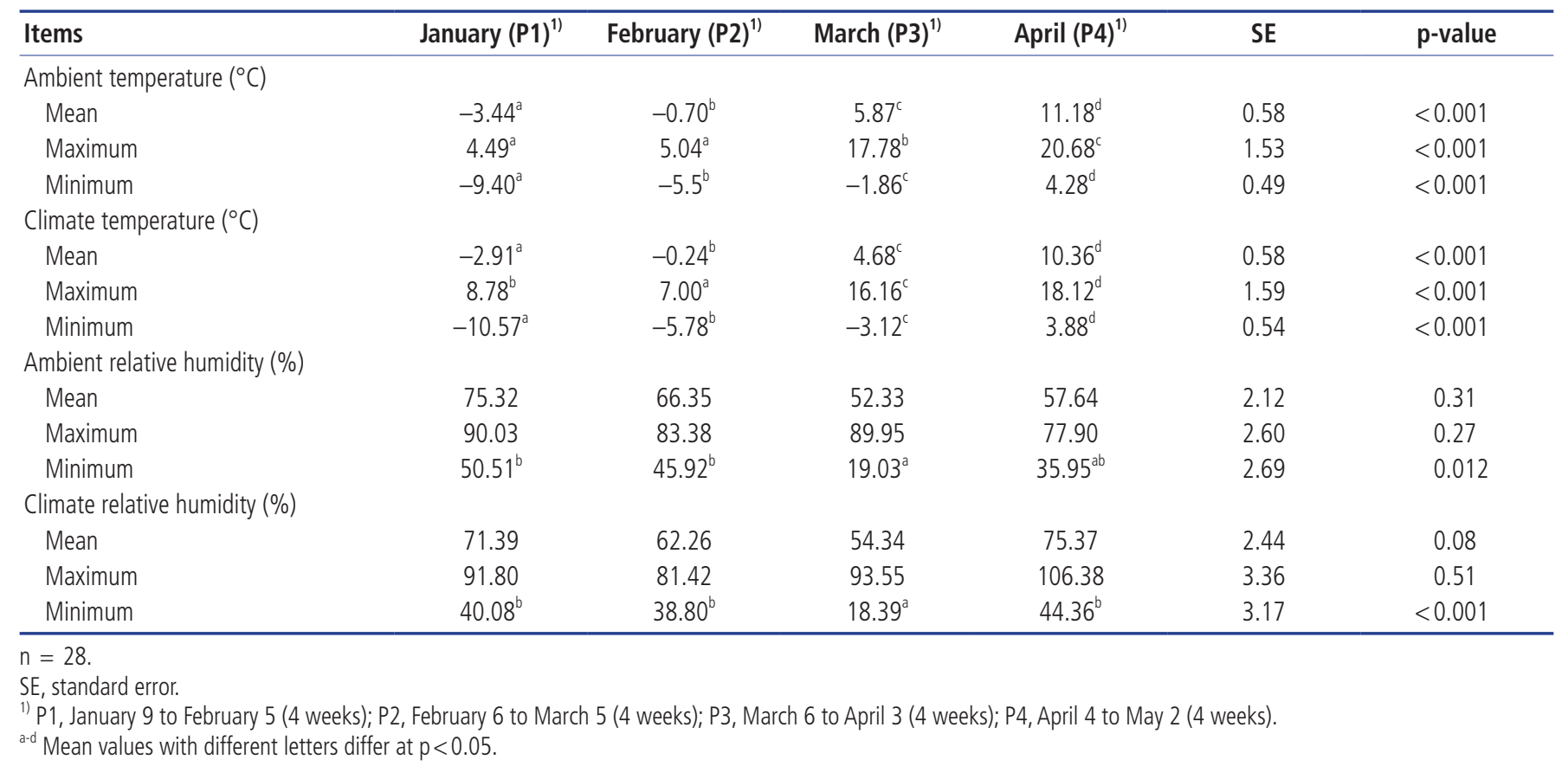


Table 3. Growth performance of Korean cattle steers fed either control or RPF-supplemented diet during January through April of 2015

\begin{tabular}{|c|c|c|c|c|c|c|c|c|c|c|c|c|}
\hline \multirow{2}{*}{ Items } & \multicolumn{2}{|c|}{ January $(\mathrm{P} 1)^{1)}$} & \multicolumn{2}{|c|}{ February $(\mathbf{P} 2)^{1)}$} & \multicolumn{2}{|c|}{$\operatorname{March}(\mathrm{P} 3)^{1)}$} & \multicolumn{2}{|c|}{ April $(\mathrm{P} 4)^{1)}$} & \multirow{2}{*}{ SE } & \multicolumn{3}{|c|}{ p-value } \\
\hline & Control & RPF & Control & RPF & Control & RPF & Control & RPF & & Month & Diet & Interaction \\
\hline Initial body weight $(\mathrm{kg})$ & 547.2 & 554.0 & 571.6 & 579.0 & 587.2 & 595.6 & 604.4 & 616.0 & 5.28 & $<0.001$ & 0.34 & 0.87 \\
\hline Body weight $(\mathrm{kg})$ & 571.6 & 579.0 & 587.2 & 595.6 & 604.4 & 616.0 & 625.2 & 636.8 & 5.57 & $<0.001$ & 0.35 & 0.94 \\
\hline \multicolumn{13}{|c|}{ Daily feed intake (kg: DM base) } \\
\hline Total feed intake $(\mathrm{kg} / \mathrm{d})$ & 7.52 & 7.43 & 7.24 & 7.38 & 7.59 & 7.51 & 7.66 & 7.76 & 0.07 & 0.12 & 0.92 & 0.75 \\
\hline Concentrate intake $(\mathrm{kg} / \mathrm{d})$ & 6.66 & 6.57 & 6.50 & 6.64 & 6.84 & 6.76 & 6.91 & 7.01 & 0.07 & 0.03 & 0.93 & 0.74 \\
\hline Forage intake (kg/d) & 0.86 & 0.86 & 0.74 & 0.74 & 0.75 & 0.75 & 0.75 & 0.75 & 0.01 & $<0.001$ & 0.98 & 0.99 \\
\hline Average daily gain $(\mathrm{kg} / \mathrm{d})$ & 0.87 & 0.89 & 0.56 & 0.59 & 0.61 & 0.73 & 0.77 & 0.77 & 0.03 & 0.39 & 0.41 & 0.62 \\
\hline Feed efficiency (G:F ratio) & 0.104 & 0.124 & 0.077 & 0.080 & 0.080 & 0.097 & 0.099 & 0.098 & 0.01 & 0.35 & 0.33 & 0.60 \\
\hline
\end{tabular}

$n=10 /$ group.

RPF, rumen-protected fat; $S E$, standard error.

1) P1, January 9 to February 5 (4 weeks): a mild cold stress range; P2, February 6 to March 5 (4 weeks): a mild cold stress range; P3, March 6 to April 3 (4 weeks): a thermo-neutral range; P4, April 4 to May 2 (4 weeks): a thermo-neutral range.

$\mathrm{NH}_{3}$ production with barley-canola seed meal and lucerne diets, but reduced $\mathrm{NH}_{3}$ production with a bromegrass diet. Meanwhile, cold exposure reduced the irreversible loss of both plasma urea and rumen $\mathrm{NH}_{3}$, and the conversion of plasma urea-nitrogen into rumen $\mathrm{NH}_{3}$ was greater in cold-exposed sheep than in warm sheep [23]. The same authors reported an increased efficiency of ruminal microbial synthesis under cold exposure in sheep. Thus, the differences in rumen $\mathrm{NH}_{3}$ concentrations between cold winter and spring in this study may have been related to changes in the irreversible loss of $\mathrm{NH}_{3}$, conversion of urea into $\mathrm{NH}_{3}$, or to the efficiency of microbial synthesis upon cold exposure. Meanwhile, RPF supplementation did not affect ruminal $\mathrm{NH}_{3}-\mathrm{N}$ concentrations.

Neither month nor RPF supplementation affected C2, C3, iso-C5, C5, or total VFA concentrations in rumen fluid ( $\mathrm{p}>$ 0.05 ; Table 4). The C2:C3 ratio tended to be lower $(\mathrm{p}=0.07)$ in the RPF supplementation group than in the control group. In another study, increasing levels of protected lipids linearly increased the molar proportion of $\mathrm{C} 3$, whereas the molar proportion of $\mathrm{C} 2$ remained unchanged, resulting in a linear decrease in the $\mathrm{C} 2: \mathrm{C} 3$ ratio [24]. $\mathrm{RPF}$ supplementation decreased $(\mathrm{p}=$ 0.02 ) ruminal iso- $\mathrm{C} 4$ concentrations, while ruminal $\mathrm{C} 4$ concentrations varied by season $(\mathrm{p}=0.02$; Table 4$)$.

\section{Blood cortisol and metabolites}

Plasma cortisol concentrations were lower $(\mathrm{p}<0.05)$ on the starting day than during the other periods, and RPF supplementation did not affect $(\mathrm{p}>0.05)$ cortisol concentrations (Table 5). For comparison, plasma cortisol and corticosterone concentrations were similar among newborn calves at $-4.0^{\circ} \mathrm{C}$ and $16^{\circ} \mathrm{C}$, although the concentrations varied at $-12^{\circ} \mathrm{C}$ and $-18^{\circ} \mathrm{C}$ in one or two animals [25]. Collectively, blood cortisol concentration does not appear to be a suitable marker of cold stress, although cortisol is commonly used as a general stress marker [26].

Serum glucose concentrations were generally higher $(\mathrm{p}<$

Table 4. Ruminal pH, VFAs and $\mathrm{NH}_{3}-\mathrm{N}$ of Korean cattle steers fed either control or RPF-supplemented diet during January through April of 2015

\begin{tabular}{|c|c|c|c|c|c|c|c|c|c|c|c|c|}
\hline \multirow{2}{*}{ Items } & \multicolumn{2}{|c|}{ February 6} & \multicolumn{2}{|c|}{ March 5} & \multicolumn{2}{|c|}{ April 3} & \multicolumn{2}{|c|}{ May 2} & \multirow{2}{*}{ SE } & \multicolumn{3}{|c|}{$p$-value } \\
\hline & Control & RPF & Control & RPF & Control & RPF & Control & RPF & & Month & Diet & Interaction \\
\hline Temperature ${ }^{1)}\left({ }^{\circ} \mathrm{C}\right)$ & \multicolumn{2}{|c|}{-0.26} & \multicolumn{2}{|c|}{6.79} & \multicolumn{2}{|c|}{10.8} & \multicolumn{2}{|c|}{14.0} & & & & \\
\hline $\mathrm{pH}$ & 7.21 & 7.04 & 6.74 & 6.50 & 6.73 & 6.77 & 6.85 & 6.90 & 0.06 & 0.46 & 0.50 & 0.17 \\
\hline $\mathrm{NH}_{3}-\mathrm{N}(\mathrm{mg} / \mathrm{dL})$ & 13.30 & 8.97 & 11.06 & 13.59 & 5.19 & 4.57 & 7.42 & 3.46 & 0.69 & $<0.001$ & 0.40 & 0.45 \\
\hline $\mathrm{C} 2(\mathrm{mM})$ & 36.22 & 37.84 & 51.24 & 52.71 & 43.89 & 46.06 & 46.19 & 39.34 & 1.12 & 0.82 & 0.87 & 0.18 \\
\hline $\mathrm{C} 3(\mathrm{mM})$ & 10.25 & 10.98 & 15.81 & 17.39 & 14.48 & 18.13 & 13.60 & 12.63 & 0.54 & 0.47 & 0.22 & 0.59 \\
\hline $\mathrm{C} 2: \mathrm{C} 3$ ratio & 3.62 & 3.46 & 3.29 & 3.11 & 3.14 & 2.84 & 3.46 & 3.14 & 0.07 & 0.24 & 0.07 & 0.66 \\
\hline iso- $\mathrm{C} 4(\mathrm{mM})$ & 0.71 & 0.65 & 0.88 & 0.81 & 0.74 & 0.71 & 0.86 & 0.68 & 0.02 & 0.63 & 0.02 & 0.22 \\
\hline $\mathrm{C} 4(\mathrm{mM})$ & 10.28 & 10.01 & 13.99 & 14.99 & 9.79 & 10.66 & 10.27 & 8.38 & 0.40 & 0.02 & 0.98 & 0.34 \\
\hline iso- $C 5(\mathrm{mM})$ & 1.10 & 1.15 & 1.51 & 1.27 & 1.19 & 1.06 & 1.36 & 0.98 & 0.05 & 0.62 & 0.08 & 0.27 \\
\hline $\mathrm{C} 5(\mathrm{mM})$ & 0.79 & 0.80 & 1.33 & 1.37 & 0.98 & 1.18 & 1.10 & 0.91 & 0.04 & 0.87 & 0.76 & 0.40 \\
\hline Total VFA (mM) & 59.35 & 61.43 & 84.76 & 88.55 & 71.07 & 77.81 & 73.38 & 62.93 & 1.86 & 0.86 & 0.84 & 0.22 \\
\hline
\end{tabular}

$n=10 /$ group. February 6 is within a mild cold stress range, and other 3 days are within a thermo-neutral range.

VFA, volatile fatty acid; RPF, rumen-protected fat; SE, standard error.

${ }^{1)}$ Average ambient temperatures of sampling times of each day. 
Table 5. Plasma cortisol and serum parameters of Korean cattle steers fed either control or RPF-supplemented diet during January through April of 2015

\begin{tabular}{|c|c|c|c|c|c|c|c|c|c|c|c|c|c|c|}
\hline \multirow{2}{*}{ Items } & \multicolumn{2}{|c|}{ January 9} & \multicolumn{2}{|c|}{ February 6} & \multicolumn{2}{|c|}{ March 5} & \multicolumn{2}{|c|}{ April 3} & \multicolumn{2}{|c|}{ May 2} & \multirow{2}{*}{ SE } & \multicolumn{3}{|c|}{$p$-value } \\
\hline & Control & RPF & Control & RPF & Control & RPF & Control & RPF & Control & RPF & & Month & Diet & Interaction \\
\hline Temperature ${ }^{11}\left({ }^{\circ} \mathrm{C}\right)$ & \multicolumn{2}{|c|}{-6.42} & \multicolumn{2}{|c|}{-0.26} & \multicolumn{2}{|c|}{6.79} & \multicolumn{2}{|c|}{10.8} & \multicolumn{2}{|c|}{14.0} & & & & \\
\hline Cortisol $(\mu \mathrm{g} / \mathrm{dL})$ & 35.6 & 37.6 & 64.7 & 62.2 & 57.0 & 41.2 & 54.3 & 54.0 & 59.0 & 64.5 & 5.68 & 0.012 & 0.55 & 0.65 \\
\hline Glucose (mg/dL) & 74.3 & 72.7 & 78.9 & 75.5 & 76.1 & 75.6 & 66.7 & 66.9 & 59.0 & 57.8 & 0.90 & $<0.001$ & 0.34 & 0.75 \\
\hline Cholesterol (mg/dL) & 170.1 & 174.6 & 147.4 & 168.3 & 156.1 & 168.5 & 154.7 & 178.4 & 152.1 & 180.9 & 3.09 & 0.78 & 0.004 & 0.21 \\
\hline Triglyceride (mg/dL) & 14.8 & 13.6 & 21.0 & 20.0 & 14.4 & 15.7 & 16.0 & 15.8 & 15.8 & 15.2 & 0.43 & 0.70 & 0.84 & 0.76 \\
\hline $\mathrm{HDL}(\mathrm{mg} / \mathrm{dL})$ & 101.7 & 103.9 & 97.7 & 105.3 & 94.3 & 102.3 & 93.8 & 102.1 & 87.8 & 92.5 & 1.39 & 0.003 & 0.03 & 0.75 \\
\hline LDL (mg/dL) & 23.7 & 21.9 & 17.3 & 18.7 & 19.1 & 19.3 & 19.3 & 21.7 & 21.4 & 25.4 & 0.61 & 0.31 & 0.51 & 0.13 \\
\hline $\operatorname{NEFA}(\mathrm{mg} / \mathrm{dL})$ & 303.8 & 283.0 & 193.0 & 271.4 & 439.2 & 374.1 & 296.4 & 308.9 & 264.6 & 280.7 & 11.39 & 0.95 & 0.97 & 0.88 \\
\hline Albumin (mg/dL) & 3.51 & 3.56 & 3.36 & 3.47 & 3.39 & 3.53 & 3.38 & 3.39 & 3.38 & 3.38 & 0.02 & 0.017 & 0.18 & 0.52 \\
\hline GOT (IU/dL) & 73.5 & 71.0 & 75.9 & 79.7 & 79.2 & 78.2 & 74.2 & 72.3 & 74.2 & 74.8 & 1.16 & 0.86 & 0.99 & 0.92 \\
\hline GPT (IU/dL) & 26.1 & 23.6 & 24.1 & 22.5 & 25.1 & 22.2 & 24.1 & 21.1 & 24.6 & 22.7 & 0.35 & 0.18 & $<0.001$ & 0.95 \\
\hline Calcium (mg/dL) & 9.69 & 9.77 & 9.47 & 9.40 & 9.37 & 9.78 & 9.30 & 9.31 & 9.34 & 9.25 & 0.04 & $<0.001$ & 0.40 & 0.58 \\
\hline Phosphorus (mg/dL) & 7.92 & 7.68 & 8.70 & 7.95 & 8.09 & 7.62 & 7.83 & 7.19 & 8.60 & 7.84 & 0.08 & 0.75 & $<0.001$ & 0.40 \\
\hline Magnesium (mg/dL) & 2.56 & 2.39 & 2.74 & 2.48 & 2.82 & 2.63 & 2.83 & 2.44 & 2.89 & 2.65 & 0.03 & $<0.001$ & $<0.001$ & 0.40 \\
\hline
\end{tabular}

$n=10 /$ group. January 9 and February 6 are within a mild cold stress range, and other 3 days are within a thermos-neutral range.

RPF, rumen-protected fat; SE, standard error; HDL, high density lipoprotein; LDL, low density lipoprotein; NEFA, non-esterified fatty acid; GOT, glutamic oxalacetic transaminase; GPT, glutamic pyruvate transaminase.

1) Average ambient temperatures of sampling times of each day.

0.001) in colder weather but were unaffected by RPF supplementation (Table 5). Young [27] suggested that increased blood glucose results from increased metabolism (e.g., metabolic rate or heart rate) under cold conditions. In humans, lipid and muscle glycogen have major roles in providing the energy required for heat production under cold exposure, whereas plasma glucose has only a minor role [28]. Meanwhile, in lactating beef cows, fat supplementation did not affect circulating glucose concentrations [29].

Month did not affect total cholesterol concentrations ( $\mathrm{p}>$ 0.05; Table 5), and serum HDL concentrations were similar among January, February, March, and early April, albeit that the concentrations in late April were comparatively lower. RPF supplementation increased both total cholesterol $(\mathrm{p}=0.004)$ and HDL concentrations ( $p=0.03$; Table 5). In Holstein calves, various types of fat supplementation increased plasma cholesterol and HDL concentrations, with no differences seen in TG and glucose concentrations, during the cold season [30]. Furthermore, dietary rumen-protected oleic acid increased blood HDL concentrations in cattle [31]. Collectively, the increased HDL concentrations observed in this and previous studies may be due to increased amounts of absorbed fat in the small intestine via RPF supplementation. Moreover, changes in posthepatic lipoprotein metabolism, including alterations in HDL concentrations, may contribute to increased cholesterol concentrations with RPF supplementation, as suggested by Duske et al [32].

Neither month nor RPF supplementation affected serum TG, LDL, or NEFA concentrations. The effects of RPF supplementation on blood NEFA concentrations in several studies of lactating dairy cows were inconsistent with our results [24,33], and showed an increase in blood NEFA concentrations with RPF supplementation. Overall, little information is available on the effects of RPF supplementation on NEFA concentrations in beef cattle.

Serum albumin concentrations were higher $(\mathrm{p}=0.017)$ on the experimental starting day in the colder month (January) than in the other months in spring, although the concentrations were unaffected by RPF supplementation ( $p>0.05$; Table 4). A previous study revealed higher serum albumin concentrations with changes in the osmotic pressure of body fluid during hotter summer periods than colder winter periods in dairy cows [33]. Thus, the seasonal variation in circulating albumin concentrations remains unclear.

Serum GOT and GPT concentrations were not affected by month, though GPT concentrations were lower $(\mathrm{p}<0.001)$ in the RPF supplementation group, which has not been reported previously (Table 5).

Month and/or RPF supplementation affected blood mineral concentrations (Table 5). Blood calcium concentrations were higher $(\mathrm{p}<0.001)$, while blood magnesium concentrations were lower $(\mathrm{p}<0.001)$, on the starting day in January compared with the spring months. In addition, the RPF supplementation group had lower $(\mathrm{p}<0.001)$ phosphorus and magnesium concentrations than the control group. In a previous study, serum magnesium levels were lower in hyperthyroid human patients [34]. Furthermore, thyroid hormone thyroxine increased under cold conditions in beef cattle [35]. Thus, the lower magnesium concentrations under cold conditions in this study may have been indirectly influenced by higher circulating thyroid hormone levels. However, changes in these mineral concentrations according to RPF supplementation have not been well studied. 


\section{CONCLUSION}

Growth performance (i.e., average daily gain and gain-to-feed ratio) remained unchanged during all studied months (January to May). Furthermore, rumen fermentation parameters, such as $\mathrm{C} 2, \mathrm{C} 3$, and total VFA concentrations, were unaffected by cold conditions and RPF supplementation, although $\mathrm{NH}_{3}-\mathrm{N}$ concentrations were altered under cold conditions. Serum glucose and HDL concentrations were generally higher under cold conditions; however, other serum lipid metabolites (e.g., TG, LDL, and NEFA) did not differ among the studied months. These results suggest that Korean cattle may not have been significantly affected by cold conditions in this study, although cold conditions partially affected rumen fermentation and blood parameters. Moreover, RPF supplementation did not affect growth performance or major rumen VFA (C2, C3, and C4) concentrations, although RPF supplementation resulted in altered cholesterol and HDL concentrations. Further research is warranted to clarify which cold conditions significantly affect the growth performance of Korean cattle.

\section{CONFLICT OF INTEREST}

We certify that there is no conflict of interest with any financial organization regarding the material discussed in the manuscript.

\section{ACKNOWLEDGMENTS}

This study was supported by grants from the Korea Institute of Planning and Evaluation for Technology in Food, Agriculture, Forestry and Fisheries (IPET) through Advanced Production Technology Development Program (118016-03-2-SB010) and through Agri-Bio Industry Technology Development Program (313020-04-4-HD030), funded by Ministry of Agriculture, Food and Rural Affairs (MAFRA).

\section{REFERENCES}

1. Ames DR, Brink DR, Willms CL. Adjusting protein in feedlot diets during thermal stress. J Anim Sci 1980;50:1-6.

2. Gulliksen SM, Lie KI, Løken T, Østerås O. Calf mortality in Norwegian dairy herds. J Dairy Sci 2009;92:2782-95.

3. Mader TL. Environmental stress in confined beef cattle. J Anim Sci 2003;81:E110-9.

4. Brouček J, Letkovičová M, Kovalčuj K. Estimation of cold stress effect on dairy cows. Int J Biometeorol 1991;35:29-32.

5. Young BA. Cold stress as it affects animal production. J Anim Sci 1981;52:154-63.

6. Kennedy PM, Christopherson RJ, Milligan LP. The effect of cold exposure of sheep on digestion, rumen turnover time and efficiency of microbial synthesis. Br J Nutr 1976;36:231-
42.

7. NRC. Effect of environment on nutrient requirements of domestic animals. Washington, DC, USA: National Academy Press; 1981.

8. Christopherson RJ, Kennedy PM. Effect of the thermal environment on digestion in ruminants. Can J Anim Sci 1983;63: 477-96.

9. Hess BW, Moss GE, Rule DC. A decade of developments in the area of fat supplementation research with beef cattle and sheep. J Anim Sci 2008;86:E188-204

10. NRC. Nutrient requirements of dairy cattle. 8th rev ed. Washington, DC, USA: National Academy Press; 2007.

11. Kronfeld DS, Donoghue S, Naylor JM, Johnson K, Bradley CA. Metabolic effects of feeding protected tallow to dairy cows. J Dairy Sci 1980;63:545-52.

12. McNamara S, Butler T, Ryan DP, et al. Effect of offering rumenprotected fat supplements on fertility and performance in springcalving Holstein-Friesian cows. Anim Reprod Sci 2003;79: 45-56.

13. Hill GM, West JW. Rumen protected fat in Kline barley or corn diets for beef cattle: digestibility, physiological, and feedlot responses. J Anim Sci 1991;69:3376-88.

14. Naik PK. Bypass fat in dairy ration - a review. Anim Nutr Feed Techol 2013;13:147-63.

15. AOAC. Official methods of analysis. 15th ed. Association of Official Analytical Chemists, Washington, DC, USA: AOAC International; 1996. p. 210-9.

16. Van Soest PJ, Robertson JB, Lewis BA. Methods for dietary fiber, neutral detergent fiber, and nonstarch polysaccharides in relation to animal nutrition. J Dairy Sci 1991;74:3583-97.

17.Shen JS, Chai Z, Song LJ, Liu ZX, Wu YM. Insertion depth of oral stomach tubes may affect the fermentation parameters of ruminal fluid collected in dairy cows. J Dairy Sci 2012;95:597884.

18. Chaney AL, Marbach EP. Modified reagents for determination of urea and ammonia. Clin Chem 1962;8:130-2.

19. Kang HJ, Lee IK, Baik M, et al. Effects of ambient temperature on growth performance, blood metabolites, and immune cell populations in Korean cattle steers. Asian-Australas J Anim Sci 2016;29:436-43.

20.Grzych M. Webinar Portal for Forestry and Natural Resources [Internet]. c2010 [Accessed Jun 26, 2014]. Cattle Stress Index Description. Available from: http://www.forestrywebinars.net/ webinars/planning-and-design-of-livestock-watering-systems/

21.Park SJ, Beak SH, Jung JDS, et al. Genetic, management, and nutritional factors affecting intramuscular fat deposition in beef cattle - a review. Asian-Australas J Anim Sci 2018;31: 1043-61.

22.Kennedy PN, Christopherson RJ, Milligan LP. Effects of cold exposure on feed protein degradation, microbial protein synthesis and transfer of plasma urea to the rumen of sheep. $\mathrm{Br}$ J Nutr 1982;47:521-35. 
23. Kennedy PM, Milligan LP. Effects of cold exposure on digestion, microbial synthesis and nitrogen transformations in sheep. Br J Nutr 1978;39:105-17.

24. Bines JA, Brumby PE, Storry JE, Fulford RJ, Braithwaite GD. The effect of protected lipids on nutrient intakes, blood and rumen metabolites and milk secretion in dairy cows during early lactation. J Agric Sci Camb 1978;91:135-50.

25. Khan MA, Dickson WM, Meyers KM. The effect of low environmental temperature on plasma corticosteroid and glucose concentrations in the newborn calf. J Endocrinol 1970;48:35563.

26. Pollard TM. Use of cortisol as a stress marker: practical and theoretical problems. Am J Hum Biol 1995;7:265-74.

27. Young BA. Temperature-induced changes in metabolism and body weight of cattle (Bos taurus). Can J Physiol Pharmacol 1975;53:947-53.

28. Haman F, Péronnet F, Kenny GP, et al. Effect of cold exposure on fuel utilization in humans: plasma glucose, muscle glycogen, and lipids. J Appl Physiol 2002;93:77-84.

29.Lake SL, Scholljegerdes EJ, Atkinson RL, et al. Body condition score at parturition and postpartum supplemental fat effects on cow and calf performance. J Anim Sci 2005;83:2908-17.

30. Ghasemi E, Azad-Shahraki M, Khorvash M. Effect of different fat supplements on performance of dairy calves during cold season. J Dairy Sci 2017;100:5319-28.

31.Lee H-J, Lee SC, Oh YG, et al. Effects of rumen protected oleic acid in the diet on animal performances, carcass quality and fatty acid composition of Hanwoo steers. Asian-Australas J Anim Sci 2003;16:1003-10.

32. Duske K, Hammon HM, Langhof AK, et al. Metabolism and lactation performance in dairy cows fed a diet containing rumenprotected fat during the last twelve weeks of gestation. J Dairy Sci 2009;92:1670-84.

33. Payne JM, Rowlands GJ, Manston R, Dew SM, Parker WH. A statistical appraisal of the results of the metabolic profile tests on 191 herds in the B.V.A.-A.D.A.S joint exercise in animal health and productivity. Br Vet J 1974;130:34-44.

34.Ebel H, Gunther T. Magnesium metabolism: a review. J Clin Chem Clin Biochem 1980;18:257-70.

35. Christopherson RJ, Gonyou HW, Thompson JR. Effects of temperature and feed intake on plasma concentration of thyroid hormones in beef cattle. Can J Anim Sci 1979;59:655-61. 